\title{
Teaching an old pain medicine society new tweets: integrating social media into continuing medical education
}

\author{
Kellie M. Jaremko ${ }^{1}$, Eric S. Schwenk ${ }^{2}$, Amy C.S. Pearson ${ }^{3}$, \\ Jonathan Hagedorn ${ }^{4}$, Ankeet D. Udani ${ }^{5}$, Gary Schwartz ${ }^{6}$, \\ Nabil M. Elkassabany ${ }^{7}$, Anne Snively ${ }^{8}$, and Edward R. Mariano, ${ }^{9,10}$ \\ ${ }^{1}$ Department of Anesthesia, Critical Care and Pain Medicine, Massachusetts General Hospital, Boston, MA, \\ ${ }^{2}$ Department of Anesthesiology, Sidney Kimmel Medical College at Thomas Jefferson University, Philadelphia, PA, \\ ${ }^{3}$ Department of Anesthesia, University of Iowa, Iowa City, IA, ${ }^{4}$ Department of Anesthesiology and Perioperative \\ Medicine, Mayo Clinic, Rochester, MN, ${ }^{5}$ Department of Anesthesiology, Duke University School of Medicine, \\ Durham, NC, ${ }^{6}$ Department of Anesthesiology, Maimonides Medical Center, Brooklyn, NY, ${ }^{7}$ Department of \\ Anesthesiology and Critical Care, University of Pennsylvania, Philadelphia, ${ }^{8}$ Director of Communications, American \\ Society of Regional Anesthesia and Pain Medicine, Pittsburgh, PA, ${ }^{9}$ Department of Anesthesiology, Perioperative \\ and Pain Medicine, Stanford University School of Medicine, Stanford, ${ }^{10}$ Anesthesiology and Perioperative Care \\ Service, Veterans Affairs Palo Alto Health Care System, Palo Alto, CA, USA
}

Today, social media has surpassed print newspapers as the primary source of news [1]. In medical education, social media has developed into a tool for disseminating new research findings and learning points from scientific conferences in real time to online audiences throughout the world [2-4]. Social media metrics are increasingly becoming used in academic appointment and promotion portfolios [5]. Twitter in particular has become the social media platform of choice for conference attendees across medical specialties [6-10], and studies show that the majority of tweets generated from conferences are educational $[10,11]$.

The current opioid epidemic represents an opportunity for

Corresponding author: Edward R. Mariano, M.D., MAS

Anesthesiology and Perioperative Care Service, VA Palo Alto Health Care System, 3801 Miranda Avenue, MC 112A, Palo Alto, CA 94304, USA

Tel: +1-650-849-0254, Fax: +1-650-852-3423

Email: emariano@stanford.edu

ORCID: https://orcid.org/0000-0003-2735-248X

Received: June 17, 2019.

Accepted: June 18, 2019.

Korean J Anesthesiol 2019 October 72(5): 409-412 https://doi.org/10.4097/kja.19261 pain medicine societies to take advantage of social media to promote the latest research and key educational messages from conferences to aid clinicians and patients. However, to date there are no published reports of social media implementation strategies from the field of chronic pain. We therefore provide our experience deliberately integrating Twitter use into the annual fall pain medicine conference hosted by the American Society of Regional Anesthesia and Pain Medicine (ASRA) and share strategies used to promote high-quality tweets and drive engagement. This project was granted exemption from review by the Institutional Review Board.

ASRA was originally founded in 1923 but dissolved by 1940 when members were encouraged to join the growing American Society of Anesthetists, which eventually became the American Society of Anesthesiologists [12]. The modern ASRA was founded again in 1975, and its current vision is to relieve the global burden of pain (https://www.asra.com/about). We conducted this comparison of Twitter use for the fall 2015 and 2016 annual pain medicine meetings of ASRA since the Society developed a social media strategy in between these two conferences. Free Twitter transcripts were obtained for \#ASRAPain15 and \#ASRAPain16; these hashtags were registered with Symplur (Pasadena, CA, USA), a healthcare social media analytics company, in advance of each meeting.

(c) This is an open-access article distributed under the terms of the Creative Commons Attribution Non-Commercial License (http://creativecommons.org/ licenses/by-nc/4.0/), which permits unrestricted non-commercial use, distribution, and reproduction in any medium, provided the original work is properly cited. 


\section{Social Media Strategy}

Prior to the 2016 ASRA spring regional anesthesia annual meeting, a new social media education and engagement strategy was implemented and directed towards members and meeting attendees [11]. No specific strategy existed prior to this year, and implementation continued for the 2016 ASRA fall pain medicine meeting. In August 2016, ASRA posted an article on its website with specific tips on writing blogs, which would also apply to other social media posts (https://www.asra.com/news/117/ tips-for-writing-blog-entries). All correspondence (e.g., emails, flyers) prior to the 2016 fall pain medicine meeting featured the hashtag \#ASRAPain16. Although the 2015 fall pain medicine meeting had a hashtag \#ASRAPain15, this was not heavily promoted. A 'Social Media Team' was identified in 2016 based on members and past meeting attendees who had been previously active on Twitter. These individuals were asked to promote the upcoming fall pain medicine meeting in advance as well as 'live tweet' [13] and actively participate in the \#ASRAPain16 Twitter conversations during the conference.

\section{Metrics}

Tweets for each of the conferences were collected as transcripts starting with registration opening on the first day of the meeting and ending on the last day of the meeting at the conclusion of the last session. Impressions (the number of tweets multiplied by the number of followers who can view the tweets) and number of Twitter participants (those who created at least one tweet) were collected from Symplur free analytics [11]. All tweets were manually counted and reviewed by two investigators independently. Among the complete list of tweets, replies (tweets in response to another Twitter user's original tweet), and retweets were differentiated from original tweets. Tweets were assigned to one of four categories (scientific, logistical, social, or other) based on a classification system described previously [11]. Specific content of tweets was assessed for the inclusion of media (e.g., images, video), external links ('for more information'), and mentions (including another Twitter user's 'handle' or username in the tweet). Analyses were performed with NCSS-PASS statistical software (NCSS, LLC, Kaysville, UT). Comparisons of proportions were conducted using the Chi square test or Fisher's Exact test if $\mathrm{n}<5$ in any field. For all analyses, $\mathrm{P}<0.05$ was considered statistically significant.

\section{\#ASRAPain15 and versus \#ASRAPain16}

The number of tweets generated by the ASRA fall pain medicine meeting increased from 148 for \#ASRAPain 15 to 312 for \#ASRAPain16. The number of impressions increased from
527,489 for \#ASRAPain15 to 1,746,713 for \#ASRAPain16, and the number of participants also increased from 128 for \#ASRAPain15 to 196 for \#ASRAPain16. Nearly all tweets were original and not replies or modified retweets: 136 (92\%) for \#ASRAPain 15 and 284 (91\%) for \#ASRAPain16 (P = 0.758). Most tweets were scientific in nature: $57 \%$ for \#ASRAPain 15 and 161 (52\%) for \#ASRAPain16 ( $\mathrm{P}=0.242$ ). Some form of media (e.g., image) was included in 92 (62\%) of \#ASRAPain15 tweets compared to $164(53 \%)$ of \#ASRAPain16 tweets $(\mathrm{P}=0.053)$. Links were included in 15 (10\%) of \#ASRAPain 15 tweets compared to 71 (23\%) of \#ASRAPain16 tweets $(\mathrm{P}=0.001)$, and at least one other Twitter user was mentioned in 63 (42\%) of \#ASRAPain 15 tweets compared to 168 (54\%) of \#ASRAPain 16 tweets ( $\mathrm{P}=$ $0.024)$. The number of tweets with at least one retweet was 65 (44\%) for \#ASRAPain15 versus 175 (56\%) for \#ASRAPain16 (P = $0.015)$.

This is the first description of a deliberate social media strategy to promote Twitter activity and engagement for an annual pain medicine conference. Despite being one of the oldest subspecialty societies in anesthesiology, ASRA has embraced technology and innovation and has made social media a point of emphasis in its communications. Following implementation of a focused strategy between the fall 2015 and 2016 annual pain medicine meetings of ASRA, the number of tweets, participants, and impressions generated as well as the content quality of tweets increased in the subsequent meeting.

Today, Twitter functions as a tool for the modern learner $[2,14]$. While the use of Twitter during scientific meetings is becoming commonplace $[10,11]$, the selection of metrics in research studies analyzing Twitter data has not yet been standardized [2]. We propose four categories of Twitter metrics for the purposes of reporting Twitter use in medical education (Fig. 1). We further suggest the following best practices for live tweeting (Table 1) [15]. Our example of one professional pain medicine society's integration of Twitter into its annual scientific conference demonstrates that a focused implementation strategy may

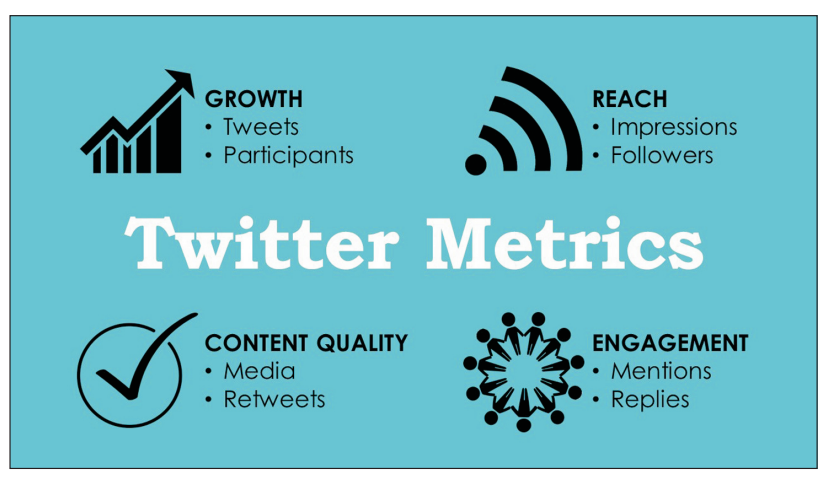

Fig. 1. Four categories of proposed metrics for the reporting of Twitter use in medical education. 
Table 1. Suggested Best Practices for Live Tweeting during a Medical Conference

1. Register the conference hashtag with Symplur before the event: https://www.symplur.com/healthcare-hashtags/. A free account gives conference organizers and planning committee members access to basic analytics and transcript services for up to 30 days after the event.

2. Advertise the conference hashtag on all promotional materials and include it in all tweets related to the conference. The hashtag allows other Twitter users to easily find tweets related to the conference since a hashtag is a live link within the body of a tweet. After the event, all tweets that include the conference hashtag can be collected and saved in the form of a transcript using Symplur.

3. Emphasize quality and not quantity of tweets. It is too difficult (and unnecessary) to give a phrase-by-phrase reproduction of a speaker's entire lecture. Remember that the primary purposes of attending a conference are to learn and network, so spend more time on these activities and not tweeting. Consider summarizing two or three salient points from a presentation into one tweet or tweeting photos with a short commentary to provide context for the Twitter community.

4. Give credit where credit is due. Before tweeting, do a little homework. Use the search function on Twitter (spyglass icon) to check if a speaker has a Twitter account; if so, include the speaker's 'handle' (username) in your tweet. If the speaker references a key article, find the link and include it in your tweet. These elements make a tweet more informative to the reader and may increase the likelihood of its being retweeted or generating further conversation on Twitter.

5. Keep tweets clean and professional. Healthy debate is one of the best parts of scientific conferences, but avoid writing anything in a tweet that would not be said in public. Above all, remember to protect patient privacy and confidentiality.

be associated with improvements in the quantity and quality of live tweets and attendee engagement.

\section{Acknowledgments}

This material is the result of work supported with resources and the use of facilities at the VA Palo Alto Health Care System (Palo Alto, CA, USA). The contents do not represent the views of VA or the United States Government.

\section{Conflicts of Interest}

No potential conflict of interest relevant to this article was reported.

\section{Author Contributions}

Kellie M. Jaremko (Conceptualization; Data curation; Formal analysis; Investigation; Methodology; Writing-original draft; Writing-review \& editing)

Eric S. Schwenk (Conceptualization; Data curation; Formal analysis; Investigation; Methodology; Writing-original draft; Writing-review \& editing)

Amy C.S. Pearson (Data curation; Investigation; Methodology; Writing-review \& editing)

Jonathan Hagedorn (Data curation; Investigation; Methodology;
Writing-review \& editing)

Ankeet D. Udani (Data curation; Investigation; Methodology; Writing-review \& editing)

Gary Schwartz (Data curation; Investigation; Methodology; Writing-review \& editing)

Nabil M. Elkassabany (Data curation; Investigation; Methodology; Writing-review \& editing)

Anne Snively (Investigation; Methodology; Project administration; Writing-review \& editing)

Edward R. Mariano (Conceptualization; Data curation; Formal analysis; Investigation; Methodology; Project administration; Resources; Software; Supervision; Validation; Visualization; Writing-original draft; Writing-review \& editing)

\section{ORCID}

Kellie M. Jaremko, https://orcid.org/0000-0001-9342-1979

Eric S. Schwenk, https://orcid.org/0000-0003-3464-4149

Amy C.S. Pearson, https://orcid.org/0000-0002-7911-3192

Jonathan Hagedorn, https://orcid.org/0000-0003-1039-8166

Ankeet D. Udani, https://orcid.org/0000-0002-6195-7151

Gary Schwartz, https://orcid.org/0000-0001-6274-1945

Nabil M. Elkassabany, https://orcid.org/0000-0003-3503-4828

Anne Snively, https://orcid.org/0000-0002-0903-6419

Edward R. Mariano, https://orcid.org/0000-0003-2735-248X

\section{References}

1. Shearer E. Social media outpaces print newspapers in the U.S. as a news source Fact Tank [Internet]. Washington: Pew Research Center; 2018 Dec [cited 2019 Apr 3]. Available from https://www.pewresearch.org/facttank/2018/12/10/social-media-outpaces-print-newspapersin-the-u-s-as-a-news-source/.

2. Schwenk ES, Chu LF, Gupta RK, Mariano ER. How social media is changing the practice of regional anesthesiology. Curr Anesthesiol Rep 2017; 7: 238-45. 
3. Wilkinson SE, Basto MY, Perovic G, Lawrentschuk N, Murphy DG. The social media revolution is changing the conference experience: analytics and trends from eight international meetings. BJU Int 2015; 115: 839-46.

4. Jalali A, Sherbino J, Frank J, Sutherland S. Social media and medical education: Exploring the potential of Twitter as a learning tool. Int Rev Psychiatry 2015; 27: 140-6.

5. Cabrera D, Vartabedian BS, Spinner RJ, Jordan BL, Aase LA, Timimi FK. More than likes and Tweets: creating social media portfolios for academic promotion and tenure. J Grad Med Educ 2017; 9: 421-5.

6. Søreide K, Mackenzie G, Polom K, Lorenzon L, Mohan H, Mayol J. Tweeting the meeting: quantitative and qualitative twitter activity during the 38th ESSO conference. Eur J Surg Oncol 2019; 45: 284-9.

7. Luc JG, Antonoff MB. Live Tweet The Society of Thoracic Surgeons Annual Meeting: How to Leverage Twitter to Maximize Your Conference Experience. Ann Thorac Surg 2018; 106: 1597-601.

8. Hong FC, Devine P, McDonald JJ, Cologne K, Brady RR. Social media engagement amongst 2017 colorectal surgery Tripartite Meeting attendees: updates on contemporary social media use. Colorectal Dis 2018; 20: O114-8.

9. Logan AC. Using social media at national meetings in hematology-optimal use, tips, strategies, and limitations. Curr Hematol Malig Rep 2017; 12: 605-10.

10. Attai DJ, Radford DM, Cowher MS. Tweeting the Meeting: Twitter Use at The American Society of Breast Surgeons Annual Meeting 20132016. Ann Surg Oncol 2016; 23: 3418-22.

11. Schwenk ES, Jaremko KM, Gupta RK, Udani AD, McCartney CJL, Snively A, et al. Upgrading a social media strategy to increase Twitter engagement during the Spring Annual Meeting of the American Society of Regional Anesthesia and Pain Medicine. Reg Anesth Pain Med 2017; 42: 283-8.

12. Mandabach MG, Wright AJ. The American Society of Regional Anesthesia: a concise history of the original group-its birth, growth, and eventual dissolution. Reg Anesth Pain Med 2006; 31: 53-65.

13. Ekins S, Perlstein EO. Ten simple rules of live tweeting at scientific conferences. PLoS Comput Biol 2014; 10: e1003789.

14. Schwenk ES, Udani AD, Gupta RK, Mariano ER. How academic physicians can benefit from social media. Rev Esp Anestesiol Reanim 2018; 65: 103-7.

15. Mariano ER. Tips for Live Tweeting a Meeting ASRA Blog [Internet]. Pittsburgh: American Society of Regional Anesthesia and Pain Medicine; 2018 Apr 6 [cited 2019 May 16]. Available from https://www.asra.com/news/192/tips-for-live-tweeting-a-meeting. 